PROCEEDINGS OF THE

AMERICAN MATHEMATICAL SOCIETY

Volume 141, Number 4, April 2013, Pages 1177-1183

S 0002-9939(2012)11453-4

Article electronically published on August 28, 2012

\title{
EVERY MODULE IS AN INVERSE LIMIT OF INJECTIVES
}

\author{
GEORGE M. BERGMAN
}

(Communicated by Birge Huisgen-Zimmermann)

\begin{abstract}
It is shown that any left module $A$ over a ring $R$ can be written as the intersection of a downward directed system of injective submodules of an injective module; equivalently, as an inverse limit of one-to-one homomorphisms of injectives. If $R$ is left Noetherian, $A$ can also be written as the inverse limit of a system of surjective homomorphisms of injectives. Some questions are raised.
\end{abstract}

The flat modules over a ring are precisely the direct limits of projective modules [11], 6], [10, Theorem 2.4.34]. Which modules are, dually, inverse limits of injectives?

I sketched the answer in 1, but in view of the limited distribution of that item, it seems worthwhile to make the result more widely available. The construction from 11 is Theorem 2 below; the connecting maps there are inclusions. In Theorem 4, we shall see that the connecting maps can, alternatively, be taken to be onto if $R$ is Noetherian on the appropriate side.

In 92 we ask some questions, in $\$ 3$ we take some steps toward answering one of them, and in $\$ 4$ we note what the proofs of our results tell us when applied to not necessarily injective modules.

Throughout, "ring" means associative ring with unit, and modules are unital.

\section{Main Results}

We will need the following generalization of the familiar observation ([4, Proposition I.3.1], 9, Proposition IV.3.7]) that a direct product of injective modules is injective. (It is a generalization because on taking $\kappa>\operatorname{card}(I)$, it yields that result.)

Lemma 1. Let $R$ be a ring, $\kappa$ an infinite regular cardinal such that every left ideal of $R$ can be generated by $<\kappa$ elements, and $\left(M_{i}\right)_{i \in I}$ a family of left $R$-modules. Let

$$
\prod_{I}^{\kappa} M_{i}=\left\{x \in \prod_{I} M_{i} \mid x \text { has support of cardinality }<\kappa \text { in } I\right\} .
$$

Then if all $M_{i}$ are injective, so is $\prod_{I}^{\kappa} M_{i}$.

Proof. To show that $\prod_{I}^{\kappa} M_{i}$ is injective, it suffices by [4, Theorem I.3.2], [9. Lemma IV.3.8] to show that for every left ideal $L$ of $R$, every module homomorphism $h: L \rightarrow \prod_{I}^{\kappa} M_{i}$ can be extended to all of $R$. By choice of $\kappa, L$ has a generating set $X$ of cardinality $<\kappa$, and by definition of $\prod_{I}^{\kappa} M_{i}$, the image under $h$

Received by the editors April 15, 2011 and, in revised form, August 16, 2011.

2010 Mathematics Subject Classification. Primary 16D50, 18A30; Secondary 13C11, 16D90.

Key words and phrases. Inverse limit of injective modules.

http://arXiv.org/abs/arXiv:1104.3173. After publication of this note, updates, errata, related references, etc., if found, will be recorded at http://math.berkeley.edu/ gbergman/papers/. 
of each member of $X$ has support of cardinality $<\kappa$ in $I$. Hence by regularity of $\kappa$, the union $I_{0} \subseteq I$ of these supports has cardinality $<\kappa$. Clearly $h(L)$ has support in $I_{0}$; hence $h$ can be looked at as a homomorphism $L \rightarrow \prod_{I_{0}} M_{i}$. As each $M_{i}$ is injective, we can now lift $h$ componentwise to a homomorphism $R \rightarrow \prod_{I_{0}} M_{i} \subseteq \prod_{I}^{\kappa} M_{i}$, as desired.

Theorem 2. Let $R$ be a ring. Then every left $R$-module $A$ can be written as the intersection of a downward directed system of injective submodules of an injective module, in other words, as the inverse limit of a system of injective modules and one-to-one homomorphisms.

Proof. Given $A$, choose an exact sequence of modules

$$
0 \rightarrow A \rightarrow M \rightarrow N
$$

with $M$ and $N$ injective, as we may by 4, Theorem I.3.3], and call the second map $f: M \rightarrow N$. Taking a cardinal $\kappa$ as in the preceding lemma (for example, any infinite regular cardinal $>|R|)$, and a set $I$ of cardinality $\geq \kappa$, one element of which we will denote by 0 , we define $R$-modules $M_{i}(i \in I)$ by letting $M_{0}=M$, and $M_{i}=N$ for $i \neq 0$.

Now let $P=\prod_{I}^{\kappa} M_{i}$, and for each finite subset $D \subseteq I-\{0\}$, let $P_{D} \subseteq P$ be the submodule of elements $\left(x_{i}\right)_{i \in I}$ such that for all $i \in D, x_{i}=f\left(x_{0}\right)$. Clearly, each element of $P_{D}$ is determined by its components at the indices in $I-D$, from which we see that $P_{D} \cong \prod_{I-D}^{\kappa} M_{i}$; so by Lemma 1 $P_{D}$ is injective. The family of submodules $P_{D}$ is downward directed, since $P_{D_{1}} \cap P_{D_{2}}=P_{D_{1} \cup D_{2}}$.

Now $\bigcap_{D} P_{D} \subseteq P$ consists of the elements $x \in P$ such that for all $i \in I-\{0\}$, $x_{i}=f\left(x_{0}\right)$. Each such $x$ is determined by its coordinate $x_{0} \in M$, but to lie in $P$, such an element must have support of cardinality $<\kappa$, which only happens if $x_{0} \in \operatorname{ker} f$. Thus, $\bigcap_{D} P_{D} \cong \operatorname{ker} f=A$.

Note that in the construction of the above proof, if $R$ is left Noetherian, then $\kappa$ can be taken to be $\aleph_{0}$, and $I$ countable; so the intersection is over the finite subsets of a countable set, giving a countably indexed inverse system. In that situation, $\prod_{I}^{\kappa} M_{i}$ is simply $\bigoplus_{I} M_{i}$, and Lemma 1 then says that the class of injective $R$ modules is closed under direct sums (a known result, [12, Proposition 2.1]. In fact, that condition is necessary and sufficient for $R$ to be left Noetherian 13 , Theorem 1], [3, Theorem 1.1], 5, Theorem 20.1], a result variously called the MatlisPapp Theorem, the Cartan-Eilenberg-Bass Theorem, and by other combinations of these names.) We shall use this closure under direct sums in the proof of our next theorem, along with the following fact.

There exists an inverse system, indexed by the first uncountable or-

dinal $\omega_{1}$, of nonempty sets $S_{\alpha}$ and surjective maps $f_{\alpha \beta}: S_{\beta} \rightarrow S_{\alpha}$ $\left(\alpha \leq \beta \in \omega_{1}\right)$, which has empty inverse limit [7], [ㅇ, §2], [2].

Again, we begin with a general lemma.

Lemma 3 (after [8, §3]; cf. [2, Corollary 8]). Suppose $\left(S_{\alpha}, f_{\alpha \beta}\right)_{\alpha \leq \beta \in \omega_{1}}$ is an inverse system of sets with the properties stated in (3), and $N$ is a left module over a ring $R$. To each $\alpha \in \omega_{1}$, let us associate the direct sum $\bigoplus_{S_{\alpha}} N$ of an $S_{\alpha}$-tuple of copies of $N$; and for $\alpha \leq \beta$, let $\varphi_{\alpha \beta}: \bigoplus_{S_{\beta}} N \rightarrow \bigoplus_{S_{\alpha}} N$ be the map sending $\left(x_{j}\right)_{j \in S_{\beta}}$ to the element $\left(y_{i}\right)_{i \in S_{\alpha}}$ with components $y_{i}=\sum_{f_{\alpha \beta}(j)=i} x_{j}$. 
Then each $\varphi_{\alpha \beta}$ is surjective, but the inverse limit of the above system is the zero module.

Sketch of proof. We imitate the argument of [8] (where $R$ was a field and $N$ was $R$ ). Surjectivity of the $\varphi_{\alpha \beta}$ is clear. Now suppose $x$ belongs to the inverse limit, and let us write its components $x^{(\alpha)} \in \bigoplus_{S_{\alpha}} N\left(\alpha \in \omega_{1}\right)$. For each $\alpha \in \omega_{1}$, let $T_{\alpha} \subseteq S_{\alpha}$ be the (finite) support of $x^{(\alpha)}$. We see that the cardinalities of the $T_{\alpha}$ are monotonically nondecreasing in $\alpha$; hence, since $\omega_{1}$ has uncountable cofinality, the supremum of those cardinalities must be finite. (Indeed, for each $n$ such that some $\left|T_{\alpha}\right|$ equals $n$, let us choose an $\alpha_{n}$ realizing this value. Then the at most countably many indices $\alpha_{n}$ have a supremum, $\alpha_{\text {sup }} \in \omega_{1}$, and the finite value $\left|T_{\alpha_{\text {sup }}}\right|$ will bound all $\left.\left|T_{\alpha}\right|.\right)$

Calling this supremum $n$, we see that the set of $\alpha \in \omega_{1}$ such that $\left|T_{\alpha}\right|=n$ is an up-set in $\omega_{1}$, and that whenever $\alpha \leq \beta$ are both in this up-set, the connecting map $f_{\alpha \beta}$ gives a bijection $T_{\beta} \rightarrow T_{\alpha}$. These $n$-element sets $T_{\alpha}$ thus lead to an $n$-tuple of elements of $\lim _{\alpha} S_{\alpha}$. But by assumption, that limit set is empty. Hence $n=0$, so all $x^{(\alpha)}$ are 0 , so $x=0$.

We can now prove

Theorem 4. Let $R$ be a left Noetherian ring. Then every left $R$-module $A$ can be written as the inverse limit of a system, indexed by $\omega_{1}$, of surjective homomorphisms of injective modules.

Proof. Again let $f: M \rightarrow N$ be a homomorphism of injective left $R$-modules with kernel $A$. Let us take the inverse system of direct sums of copies of $N$ described in Lemma 3. and append to each of these direct sums a copy of $M$, getting modules

$$
M \oplus \bigoplus_{S_{\alpha}} N \quad\left(\alpha \in \omega_{1}\right),
$$

which we connect using maps that act on $M$ as the identity, and act on the direct sums of copies of $N$ by the connecting morphisms of Lemma 3 Assuming for notational convenience that none of the $S_{\alpha}$ contains an element named 0 , let us write the general element of (4) as $\left(x_{i}\right)_{i \in\{0\} \cup S_{\alpha}}$, where $x_{0} \in M$ and the other components are in $N$.

We now define, for each $\alpha \in \omega_{1}$,

$$
P_{\alpha}=\left\{x=\left(x_{i}\right)_{i \in\{0\} \cup S_{\alpha}} \in M \oplus \bigoplus_{S_{\alpha}} N \mid \sum_{i \in S_{\alpha}} x_{i}=f\left(x_{0}\right)\right\} .
$$

Note that for each $\alpha$, if we choose any $i_{0} \in S_{\alpha}$, then we can specify an element $x \in P_{\alpha}$ by choosing its components other than $x_{i_{0}}$ to comprise an arbitrary member of $M \oplus \bigoplus_{S_{\alpha}-\left\{i_{0}\right\}} N$. The value of $x_{i_{0}}$ will then be determined by the relation $\sum_{i \in S_{\alpha}} x_{i}=f\left(x_{0}\right)$. Thus, $P_{\alpha} \cong M \oplus \bigoplus_{S_{\alpha}-\left\{i_{0}\right\}} N$, a direct sum of injectives, so since $R$ is left Noetherian, each $P_{\alpha}$ is injective. Clearly, the inverse system of surjective maps among the modules (4) induces an inverse system of surjective maps among the submodules (5).

In a member of $\varliminf_{\omega_{1}} P_{\alpha}$, the $\bigoplus_{S_{\alpha}} N$-components, as $\alpha$ ranges over $\omega_{1}$, will form a member of the inverse limit of the system of Lemma 3 hence these components must all be zero. Thus, the corresponding $M$-components must belong to ker $f=A$. Since the connecting maps on these components are the identity map of $M$, the inverse limit is $A \subseteq M$. 
(Incidentally, Theorem 2 or 4 yields a correct proof of [14, Lemma 3], the statement that $\mathbb{Z}$ is an inverse limit of injective abelian groups. The construction of [14] is similar to our proof of Theorem 2, but since the groups $H_{j}$ used there are uniquely $p$-divisible for all odd primes $p$, their intersection is $p$-divisible, and so is not $\mathbb{Z}$.)

For further examples of unexpectedly small inverse limits, see [2], 7], 8], 15]. Some questions about these are noted in [2, $\S \S 4-5]$.

\section{Questions}

Theorem 4 leaves open

Question 5. For non-left-Noetherian $R$, which left $R$-modules are inverse limits of systems of surjective maps of injective $R$-modules? (All?) Does the answer change if we restrict ourselves to systems indexed, as in Theorem 4 , by $\omega_{1}$ ?

We noted following Theorem 2 that for $R$ Noetherian, the construction used there involved a countable inverse system. This suggests

Question 6. For non-left-Noetherian $R$, which left $R$-modules are inverse limits of countable systems of one-to-one maps of injective $R$-modules? (All?)

On the other hand, the construction of Theorem 4 used uncountable inverse systems in all cases, and so leaves open

Question 7. For a (left Noetherian or general) ring $R$, which left $R$-modules are inverse limits of countable systems of surjective maps of injective left $R$-modules?

\section{Partial Results on Question 7}

The answer to Question 7 cannot be either "all modules" or "only the injectives", even for $R=\mathbb{Z}$, as will be shown by Corollary 9 and Example 10, respectively.

In describing inverse limits, we have indexed our inverse systems so that the connecting maps go from higher- to lower-indexed objects. In direct limits, which appear beside inverse limits in the following preparatory lemma, we shall take the connecting maps to go from lower- to higher-indexed objects. (Thus, in each kind of limit, our index-sets are upward directed.)

Lemma 8. Let $R$ be a ring. Let $M$ be the inverse limit of a countable system of injective left $R$-modules $M_{\alpha}$ and surjective homomorphisms $\varphi_{\alpha \beta}: M_{\beta} \rightarrow M_{\alpha}(\alpha \leq$ $\beta, \alpha, \beta \in I)$, and let $N$ be the direct limit of a countable system of projective left $R$-modules $N_{\gamma}$ and one-to-one homomorphisms $\psi_{\delta \gamma}: N_{\gamma} \rightarrow N_{\delta}(\gamma \leq \delta, \gamma, \delta \in J)$.

Then any homomorphism

$$
f: N_{\gamma} \rightarrow M_{\alpha}, \text { where } \gamma \in J, \alpha \in I
$$

can be factored

$$
N_{\gamma} \rightarrow N \rightarrow M \rightarrow M_{\alpha}
$$

where the first and last maps are the canonical ones associated with the given direct and inverse limits (and the indices $\gamma$ and $\alpha$ ), while the middle map is an arbitrary module homomorphism. 
Proof. Let us be given a homomorphism (6).

Recall that every countable directed partially ordered set (or more generally, any directed partially ordered set of countable cofinality) has a cofinal chain isomorphic to $\omega$, and that a direct or inverse limit over the original set is isomorphic to the corresponding construction over any such chain. In our present situation, we can clearly take such a chain in $I$ which begins with the index $\alpha$ of (6), and such a chain in $J$ beginning with the index $\gamma$. Hence, replacing the two given systems with the systems determined by these chains, we may assume that our direct and inverse system are both indexed by $\omega$, and name the map we wish to extend $f_{0}: N_{0} \rightarrow M_{0}$ (see (8) below).

Using the projectivity of $N_{0}$ and the surjectivity of $\varphi_{01}: M_{1} \rightarrow M_{0}$, we can now factor $f_{0}$ as $\varphi_{01} g_{0}$ for some homomorphism $g_{0}: N_{0} \rightarrow M_{1}$, and then, similarly using the injectivity of $M_{1}$ and one-one-ness of $\psi_{10}: N_{0} \rightarrow N_{1}$, factor $g_{0}$ as $f_{1} \psi_{10}$ for some $f_{1}: N_{1} \rightarrow M_{1}$. Thus, we get $f_{0}=\varphi_{01} f_{1} \psi_{10}$.

We now iterate this process, getting $f_{2}: N_{2} \rightarrow M_{2}$, etc., where each composite $N_{i-1} \rightarrow N_{i} \rightarrow M_{i} \rightarrow M_{i-1}$ is the preceding map $f_{i-1}$ :

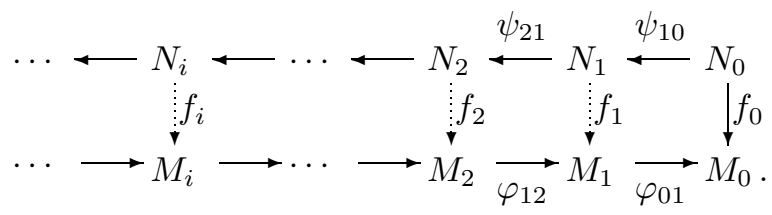

In particular, each composite $N_{0} \rightarrow N_{i} \rightarrow M_{i} \rightarrow M_{0}$ is our original map $f_{0}$. Using the universal properties of direct and inverse limits, we see that these maps induce a map $N \rightarrow M$ such that the composite $N_{0} \rightarrow N \rightarrow M \rightarrow M_{0}$ is $f_{0}$, as required.

Now suppose that $R$ is a commutative principal ideal domain. Then it is easy to verify that an $R$-module $M$ is injective if and only if it is divisible, i.e., if and only if it is a homomorphic image, as an $R$-module, of some $K$-module, where $K$ is the field of fractions of $R$. If, further, $R \neq K$ and $R$ has at most countably many primes, say $p_{1}, p_{2}, \ldots$ (where we allow repetitions in this list, in case $R$ has only finitely many), then $K$ is, as an $R$-module, the direct limit of a chain of inclusions of free $R$-modules of rank 1

$$
R \subseteq p_{1}^{-1} R \subseteq p_{1}^{-2} p_{2}^{-2} R \subseteq \cdots \subseteq p_{1}^{-i} p_{2}^{-i} \ldots p_{i}^{-i} R \subseteq \cdots
$$

Hence we can apply Lemma 8 with $K$ as $N$, calling the modules of (9) $N_{0} \subseteq N_{1} \subseteq$ $\cdots$, but still letting $\left(M_{\alpha}\right)_{\alpha \in I}$ be an arbitary countable inverse system of injectives. For any $\alpha \in I$, every $x \in M_{\alpha}$ is, of course, the image of the generator $1 \in R=N_{0}$ under some homomorphism $f: N_{0} \rightarrow M_{\alpha}$. Hence Lemma 8 tells us that $x$ lies in the image of a homomorphism $K=N \rightarrow M \rightarrow M_{\alpha}$, so the span in $M$ of the images of all homomorphisms $K \rightarrow M$ maps surjectively to each $M_{\alpha}$. For brevity and concreteness, we state this result below for $R=\mathbb{Z}$.

Corollary 9. Let $M$ be the inverse limit of a countable system of injective $\mathbb{Z}$ modules $M_{\alpha}$ and surjective homomorphisms $\varphi_{\alpha \beta}: M_{\beta} \rightarrow M_{\alpha}$. Let $M_{\mathrm{div}}$ be the largest divisible (equivalently, injective) submodule of $M$, namely, the sum of the images of all $\mathbb{Z}$-module homomorphisms $\mathbb{Q} \rightarrow M$. Then $M_{\mathrm{div}}$ projects surjectively to each $M_{\alpha}$; i.e., the composite maps $M_{\mathrm{div}} \hookrightarrow M \rightarrow M_{\alpha}$ are surjective. 
This shows that if $M$ is nontrivial, it must have a sizable injective submodule. (In particular, $M$ cannot be a nonzero finitely generated $\mathbb{Z}$-module.) However, the following example shows that this submodule need not be all of $M$.

Example 10. A countable inverse system $\cdots \rightarrow M_{2} \rightarrow M_{1} \rightarrow M_{0}$ of injective $\mathbb{Z}$-modules and surjective homomorphisms, whose inverse limit $M$ is not injective.

Construction and proof. For each $n \geq 0$, let

$$
M_{n}=\mathbb{Q} \oplus \ldots \oplus \mathbb{Q} \oplus(\mathbb{Q} / \mathbb{Z}) \oplus \ldots \oplus(\mathbb{Q} / \mathbb{Z}) \oplus \ldots,
$$

where the summands $\mathbb{Q}$ are indexed by $i=0, \ldots, n-1$, and the $\mathbb{Q} / \mathbb{Z}$ by the $i \geq n$. Define connecting maps $\varphi_{m n}: M_{n} \rightarrow M_{m}(m \leq n)$ to act componentwise, namely, as the identity map of $\mathbb{Q}$, respectively, of $\mathbb{Q} / \mathbb{Z}$, on the components with indices $i<m$ or $i \geq n$, and as the quotient map $\mathbb{Q} \rightarrow \mathbb{Q} / \mathbb{Z}$ on the $n-m$ intermediate components.

It is not hard to verify that the inverse limit $M$ of these modules can be identified with the submodule of $\mathbb{Q}^{\omega}$ consisting of those elements all but finitely many of whose components lie in $\mathbb{Z}$. (Given $x \in M$, its image in $M_{0}$ will have all but finitely many components $0 \in \mathbb{Q} / \mathbb{Z}$, and these zero components will correspond to the components of $x$ which lie in $\mathbb{Z}$.)

If we take an element $x \in M$ and a positive integer $k$ such that the entries of $x$ in $\mathbb{Z}$ are not almost all divisible by $k$, then $x$ is not divisible by $k$ in $M$. Hence $M$ is not a divisible group, i.e., is not injective.

Returning to Corollary 9, we remark that its method of proof, applied to a countable inverse limit $M$ of injective modules and surjective homomorphisms over any integral domain $R$, shows that $M$ contains many "highly divisible" elements. For most $R$, this shows that not all $R$-modules can occur as such inverse limits.

\section{Not NeCESSARILY inJeCtive MOdUleS}

None of the constructions we have used to get an inverse system of modules from an exact sequence $0 \rightarrow A \rightarrow M \rightarrow N$ are limited to the case where $M$ and $N$ are injective. Let us record what they give us in general.

Corollary 11 (to proofs of Theorems 2 and 4, and Example 10). Let $R$ be a ring, $\mathbf{M}$ a class of left $R$-modules, $\kappa$ an infinite regular cardinal such that $\mathbf{M}$ is closed under $\kappa$-restricted direct products $\prod_{I}^{\kappa} M_{\alpha}$, and $0 \rightarrow A \rightarrow M \rightarrow N$ any exact sequence of left $R$-modules with $M, N \in \mathbf{M}$. Then

(a) $A$ can be written as the inverse limit of a system of modules in $\mathbf{M}$ and one-to-one homomorphisms.

(b) If $\kappa=\aleph_{0}$ (so that the hypothesis on $\mathbf{M}$ is that it is closed under direct sums), then $A$ can be written as the inverse limit of an $\omega_{1}$-indexed system of modules in $\mathbf{M}$ and surjective homomorphisms.

(c) If, again, $\kappa=\aleph_{0}$, then the submodule of $M^{\omega}$ consisting of those elements with all but finitely many components in $A$ can be written as the inverse limit of a countable system of modules in $\mathbf{M}$ and surjective homomorphisms.

So, for instance, by (b), for any ring $R$, any $R$-module which can be written as the kernel of a homomorphism of projective modules can also be written as the inverse limit of a system of projective modules and surjective homomorphisms. 


\section{ACKNOWLEDGEMENTS}

The author is indebted to Pace Nielsen for pointing out the need to assume that $\kappa$ is regular in Lemma 1, and to the referee for some useful suggestions.

\section{REFERENCES}

[1] George M. Bergman, Final Examination, Math 250B, U.C. Berkeley, 15 May, 1990. Readable at http://math.berkeley.edu/ gbergman/grad.hndts/90Sp_250B_FE.pdf .

[2] George M. Bergman, Some empty inverse limits, unpublished note, Oct. 2005, revised July 2011. Readable at http://math.berkeley.edu/ gbergman/papers/unpub .

[3] Hyman Bass, Injective dimension in Noetherian rings, Trans. Amer. Math. Soc. 102 (1962) 18-29. MR0138644 (25:2087)

[4] Henri Cartan and Samuel Eilenberg, Homological algebra, Princeton University Press, 1956, reprinted 1999. MR1731415 (2000h:18022)

[5] Carl Faith, Algebra. II. Ring theory, Grundlehren der mathematischen Wissenschaften, No. 191. Springer, 1976. MR0427349 (55:383)

[6] V. E. Govorov, On flat modules (Russian), Sibirsk. Mat. Ž. 6 (1965) 300-304. MR.0174598 (30:4799)

[7] Leon Henkin, A problem on inverse mapping systems, Proc. Amer. Math. Soc. 1 (1950) 224-225. MR0035006 (11:675e)

[8] Graham Higman and A. H. Stone, On inverse systems with trivial limits, J. London Math. Soc. 29 (1954) 233-236. MR0061086(15:773b)

[9] Thomas W. Hungerford, Algebra, Holt, Rinehart and Winston, 1974; reprinted as Springer Graduate Texts in Math., 73, 1980. MR600654 (82a:00006)

[10] T. Y. Lam, Lectures on modules and rings, Springer Graduate Texts in Math., vol. 189, 1999. MR1653294(99i:16001)

[11] Daniel Lazard, Sur les modules plats, C. R. Acad. Sci. Paris 258 (1964) 6313-6316. MR0168625 (29:5883)

[12] Eben Matlis, Injective modules over Noetherian rings, Pacific J. Math. 8 (1958) 511-528. MR0099360 (20:5800)

[13] Zoltán Papp, On algebraically closed modules, Publ. Math. Debrecen 6 (1959) 311-327. MR0121390 (22:12128)

[14] L. Salce, Classi di gruppi abeliani chiuse rispetto alle immagini omomorfe ed ai limiti proiettivi, Rend. Sem. Mat. Univ. Padova 49 (1973) 1-7. MR.0357653 (50:10121)

[15] William C. Waterhouse, An empty inverse limit, Proc. Amer. Math. Soc. 36 (1972) 618. MR0309047 (46:8158)

Department of Mathematics, University of California, Berkeley, California 947203840

E-mail address: gbergman@math.berkeley.edu 